\title{
Optical properties of dilute nitrogen GalnNAs quantum dots
}

\author{
Stanko Tomića) \\ Computational Science and Engineering Department, CCLRC Daresbury Laboratory, Warrington, Cheshire \\ WA4 4AD, United Kingdom
}

(Received 25 December 2006; accepted 15 February 2007; published online 21 March 2007)

\begin{abstract}
The authors present a theoretical study of the ground state optical transition matrix element in quantum dots (QDs) with a dilute amount of nitrogen. They have investigated the interplay between the nitrogen to the conduction band mixing and piezoelectric field on the optical matrix element. With a reduced amount of indium and an increased amount of nitrogen in the QD, the optical matrix element becomes on the average larger and less sensitive to the variation of both the QD shape and size than is the case of an InNAs QD. The optical characteristics at room temperature and $1.5 \mu \mathrm{m}$ wavelength are discussed. (C) 2007 American Institute of Physics. [DOI: 10.1063/1.2715096]
\end{abstract}

The GaInNAs semiconductor quantum dots (QDs) with dilute amount of nitrogen substitutional impurities are promising candidates for the active region in the next generation of optoelectronic devices. ${ }^{1-5}$ It has been found that replacing a small amount of the group $\mathrm{V}$ element by nitrogen in a III-V compound reduces the energy gap ${ }^{6}$ and increases the electron effective mass, a trend that is quite opposite to the most common III-V compounds, thus offering an alternative route to band structure engineering and improved optoelectronic properties. ${ }^{7}$ Recent advances in growth techniques facilitate the fabrication of self-assembled QDs with a very small amount (less than 5\%) of nitrogen substitutional impurities in the QD region or in the capping layer. ${ }^{8-18}$ With appropriate tailoring of the QD morphology, this opens the possibility of GaAs-based optoelectronic devices emitting at $1.55 \mu \mathrm{m}$ and beyond.

The aim of this letter is to identify the factors determining the optical properties of ideal dilute nitrogen QD, its dependence on the QD shape, and size as well as on the interplay between the nitrogen to conduction band mixing and piezoelectric field. In our analysis we ignore any nitrogen atoms spatial inhomogeneity inside the QD.

The strong interaction between the $\mathrm{N}$ resonant states and the conduction band edge means that the conventional eightband $\mathbf{k} \cdot \mathbf{p}$ method $^{19,20}$ cannot be applied directly to (Ga,In)NAs and related heterostructures. Improved agreement was found between electron in-plane effective mass $^{21,22}$ as predicted by the band-anticrossing (BAC) $\operatorname{model}^{6}$ and that observed experimentally when there are no cluster states nearby in energy, ${ }^{23,24}$ with which the GaInAs conduction band (CB) edge can interact. Thus the electronic structure and optical properties are calculated below by extending the conventional eight-band $\mathbf{k} \cdot \mathbf{p}$ Hamiltonian to a ten-band model, ${ }^{25,26}$ where additional two spin degenerate N-related bands on the average represent the influence of the nitrogen. Full details of the calculations are given in Ref. 25. From non-selfconsistent wave functions, we calculate the dimensionless scaled optical matrix element, ${ }^{20} I_{[x y z]}=\left(\hbar / m_{0} P\right)^{2}\left|\hat{e} \cdot \mathbf{p}_{i, j}\right|^{2}$ $=\left|M_{i, j}\right|^{2} / P^{2}$, where $\hat{e}$ is the light polarization vector, $\mathbf{p}_{i, j}$ is the momentum operator, $M_{i, j}$ is the electron-hole overlap matrix element, and $P$ is the bulk interband momentum matrix element of the QD material.

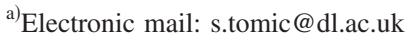

It has been shown that in the conventional InAs/GaAs QD's (Ref. 27) the optical matrix element initially increases with QD size as the electron and hole wave functions become more localized in $k$ space, providing a better overlap with highly anisotropic (at larger $k$ ) bulk optical matrix element. The QD optical matrix element then reaches a maximum for some intermediate "critical" size, beyond which it decreases in larger dots. In larger dots the piezoelectric field produces significant potential "pockets" in the QD bottom corners, in which the hole ground state can be localized. In this case the overlap with the electron wave function is significantly reduced.

In dilute nitrogen QDs two additional phenomena need to be taken into consideration. First, the presence of nitrogen increases confinement of the QD electron wave functions compared to equivalent $\mathrm{N}$-free structures. ${ }^{25}$ This means that the ground state electron wave function of the N-containing $\mathrm{QD}$, of the same size as its $\mathrm{N}$-free counterpart, is spread over larger area in $\mathbf{k}$ space, overlapping the highly anisotropic (in k space) bulk optical matrix element, $d_{i j}(\mathbf{k}){ }^{27}$ Moreover, it has been shown that the strong $\mathrm{N}-\mathrm{CB}$ mixing reduces the bulk or quantum well optical matrix element at the band edge. ${ }^{26}$ In N-containing bulk, though, the significant decrease of $\left|d_{e, h h}(\mathbf{k})\right|^{2}$ occurs at smaller wave vector than in equivalent $\mathrm{N}$-free material, ${ }^{28}$ when the electron's kinetic energy approaches the energy gap which is also reduced in $\mathrm{N}$-containing materials due to the BAC effect. Therefore, above analysis suggests the increased electron confinement effect in dilute N-containing QD implies: (i) a significantly reduced ground state optical matrix element in N-containing QDs of small to intermediate size, where confinement effects dominate over the piezoelectric field effect, (ii) a less steep initial increase of the optical matrix element as the QD size increases, due to a significant $(\sim 30 \%)$ admixture of the nitrogen character $\left|s_{\mathrm{N}}\right\rangle$ in the electron wave function, ${ }^{25}$ that provides a reduced sensitivity of the optical matrix element on the QD size, and (iii) the "critical" size of the $\mathrm{N}$-containing QD, where the optical matrix element reaches its maximum, is larger than in the N-free QDs. Secondly, the presence of nitrogen reduces the lattice mismatch of the QD material relative to the GaAs matrix. Reduced lattice mismatch reduces strain, which in turn reduces the piezoelectric field in N-containing QD structures. Being more pronounced for larger QD's, after a certain QD size, the amplified piezoelectric effect eventually dominates over the confinement 
effect. ${ }^{27}$ The reduced piezoelectric field in N-containing QDs is less able to stretch or even to confine the hole ground state wave function in piezopotential pockets. This explains why the decrease of the optical matrix element is less abrupt and occurs for larger overall critical QD sizes in N-containing than in N-free QDs. Overall, this analysis suggests that nitrogen (a) reduces the ground state optical matrix element, when compared to the same size $\mathrm{N}$-free QD, and (b) reduces the sensitivity of the optical matrix element on the QD size, providing more flexibility in device design.

To proceed with our analysis of more realistic QD structures, we consider the truncated cone QD shape. The QD size and shape can be described by three independent parameters; the QD height $h$, the aspect ratio $\alpha=h / D_{b}$, and truncation factor $t=1-D_{t} / D_{b}$, where $D_{b}$ and $D_{t}$ are diameters of the truncated cone at the base and at the truncation height, respectively. For a full cone we have $t=1$, while $t=0$ for a QD cylinder.

We first investigated the influence of the QD shape on the ground state transition energy and on the magnitude of the optical matrix element for $\hat{e}_{x}$ light polarization. To identify the influence of the In content on the electronic and optical characteristics we considered two QD materials with the same $\mathrm{N}$ content: $\mathrm{Ga}_{0.3} \mathrm{In}_{0.7} \mathrm{~N}_{0.02} \mathrm{As}_{0.98}$ and $\mathrm{InN}_{0.02} \mathrm{As}_{0.98}$. For $\mathrm{Ga}_{0.3} \mathrm{In}_{0.7} \mathrm{~N}_{0.02} \mathrm{As}_{0.98}$ QD grown at $450{ }^{\circ} \mathrm{C}$, Nishikawa et al. reported lateral dimension of the dot $D_{b}=15 \mathrm{~nm}$, height $h=5 \mathrm{~nm},{ }^{9,11}$ and PL emission $1.145 \mu \mathrm{m}$ at $T=10 \mathrm{~K} .{ }^{9}$ It has been reported by Sopasen et al. ${ }^{8}$ that the QD islands are generally bigger and of smaller density in $\operatorname{InN}_{0.02} \mathrm{As}_{0.98}$ samples than in the $\mathrm{Ga}_{0.3} \mathrm{In}_{0.7} \mathrm{~N}_{0.02} \mathrm{As}_{0.98}$ system. For the $\mathrm{InN}_{0.02} \mathrm{As}_{0.98} \mathrm{QD}$ structures the optimal growth temperature is in the range below $420{ }^{\circ} \mathrm{C}$, where its height is estimated to be $h \approx 6.7 \mathrm{~nm},{ }^{8}$ and we assume $D_{b}=20 \mathrm{~nm}$. Having fixed the aspect ratio to $\alpha=1 / 3$ and $D_{b}=15 \mathrm{~nm}$ for $\mathrm{Ga}_{0.3} \mathrm{In}_{0.7} \mathrm{~N}_{0.02} \mathrm{As}_{0.98}$ QDs, we change the truncation factor to match the $e 0-h 0$ transition wavelength with the measured one. In Fig. 1(a) the variation of the $e 0-h 0$ transition wavelength with truncation factor $t$ is presented for both material systems at low temperature and room temperature (RT). For $\mathrm{Ga}_{0.3} \mathrm{In}_{0.7} \mathrm{~N}_{0.02} \mathrm{As}_{0.98}$ QDs, under the assumptions above, we deduce that the most likely value is $t=0.8$. From the variation of the $t$ factor we estimate that QD shape inhomogeneity might introduce $\sim 10 \mathrm{meV}$ line broadening in both material systems considered. In Fig. 1(b) we present the variation of the optical matrix elements with QD shape. Due to a reduced strain in $D_{b}=15 \mathrm{~nm} \mathrm{Ga}{ }_{0.3} \mathrm{In}_{0.7} \mathrm{~N}_{0.02} \mathrm{As}_{0.98}$ QDs the effect of the piezoelectric field is significantly reduced. A larger electron-hole overlap in this system, regardless of the QD shape, is visible in Fig. 1(c). This also underlines a good uniformity of the ground state optical matrix elements. The variation in the optical matrix element is estimated to be between $+18 \%$ and $-12 \%$ of its average value of $I_{[100]}$ $=0.127$. In the indium rich, and bigger, $D_{b}=20 \mathrm{~nm}$, $\mathrm{InN}_{0.02} \mathrm{As}_{0.98}$ QDs the increased lattice mismatch produces a stronger piezoelectric field. At the same time, the increased In content moves the $\mathrm{CB}$ edge of the host material downwards on the absolute energy scale, further away from $\mathrm{N}$ resonant level, reducing the amount of $\mathrm{N}$-band character in the $e 0$ state $\left(\left|s_{\mathrm{N}}\right\rangle \sim 7 \%\right)$, indicating a larger optical matrix elements in this QD system. This is indeed visible in Fig. 1(b), for a cylindrical QD where the piezoeffect is not so influential. Away from $t=0$, the amplified piezofield has the ability to stretch the hole ground state wave function along Downloaded 22 Mar 2007 to 148.79.162.143. Redistribution subje
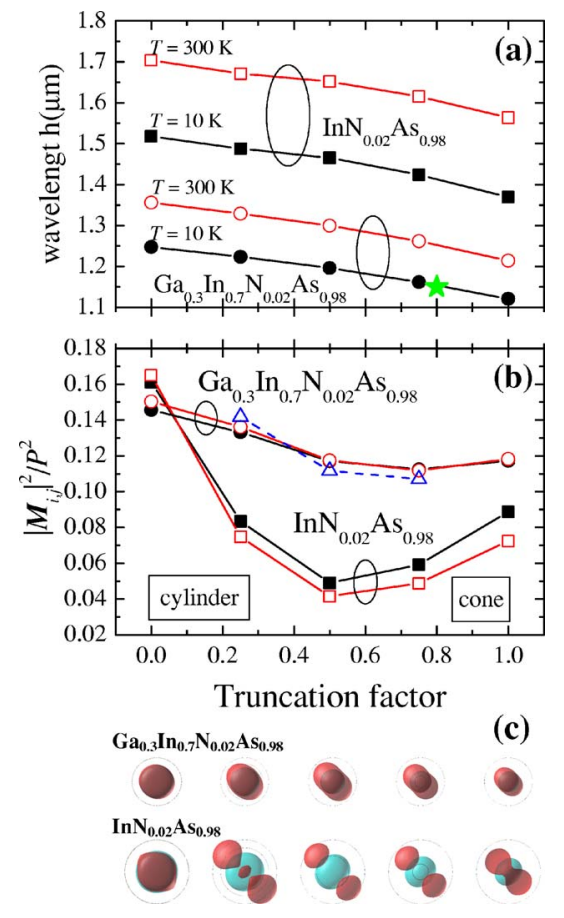

FIG. 1. (Color online) (a) QD shape variation of the $e 0-h 0$ transition wavelength for $\mathrm{Ga}_{0.3} \mathrm{In}_{0.7} \mathrm{~N}_{0.02} \mathrm{As}_{0.98}$ (circle) and $\mathrm{InN}_{0.02} \mathrm{As}_{0.98}$ (squares) at two temperatures: $T=300 \mathrm{~K}$ (open) and $T=10 \mathrm{~K}$ (solid). Star presents experimentally observed transition energy as reported in Ref. 9. (b) Variation of the optical matrix element with the QD shape in two QD material systems at two temperatures as in (a). Open triangles show the variation of the matrix element in $\mathrm{InN}_{0.02} \mathrm{As}_{0.98}$ when the piezofield is set to zero. (c) The ground state electron and hole charge density and evolution of its overlap with the QD shape change: (upper row) $\mathrm{Ga}_{0.3} \mathrm{In}_{0.7} \mathrm{~N}_{0.02} \mathrm{As}_{0.98}$ QDs and (lower row) $\mathrm{InN}_{0.02} \mathrm{As}_{0.98}$ QDs.

the $[1 \overline{1} 0]$ direction and reduce its overlap with the electron ground state. For the QDs with a $t$ factor in the range from 0.25 to 0.75 , it is estimated that the reduction of the optical matrix element due to piezoeffect is between $41 \%$ and $56 \%$. The average optical matrix element at RT is estimated to be $I_{[100]}=0.081$, with variation of $+105 \% /-48 \%$ between its maximal and minimal values. In Fig. 2(c) the reduced electron-hole overlap for a $t$ factor between 0.25 and 0.75 is visible for $\mathrm{InN}_{0.02} \mathrm{As}_{0.98}$ QDs. This analysis confirms the previous experimentally motivated suggestions that GaInNAs QDs appear more promising than InNAs QDs because more intense RT-PL is observed from GaInNAs samples. ${ }^{8}$ This is true for the QDs grown and compared at the same temperature, but a more important question would be to estimate which material will exhibit better optical characteristics at the required wavelength, for instance, 1.3 or $1.5 \mu \mathrm{m}$.

To address this question, we proceed with the analysis of the ground state emission wavelength and optical matrix element dependence on the QD size. To identify the relative role of nitrogen in that context we extend the analysis to three material systems: $\mathrm{Ga}_{0.3} \mathrm{In}_{0.7} \mathrm{~N}_{0.04} \mathrm{As}_{0.96}$, $\mathrm{Ga}_{0.3} \mathrm{In}_{0.7} \mathrm{~N}_{0.02} \mathrm{As}_{0.98}$, and $\operatorname{InN}_{0.02} \mathrm{As}_{0.98}$. We assume the same QD shape, keeping constant the truncation factor $t=0.8$ and the aspect ratio $\alpha=1 / 3$, and varying the cone base diameter in the range $D_{b} \in(10,30) \mathrm{nm}$. The $1.5 \mu \mathrm{m}$ wavelength is achievable in all three material systems, Fig. 2, although with different QD sizes: $D_{b}=28.2 \mathrm{~nm}$ for $(\mathrm{In}, \mathrm{N})$ $=(70 \%, 2 \%), D_{b}=19.2 \mathrm{~nm}$ for $(\mathrm{In}, \mathrm{N})=(70 \%, 4 \%)$, and $D_{b}=16.5 \mathrm{~nm}$ for $(\mathrm{In}, \mathrm{N})=(100 \%, 2 \%)$. In Fig. 2(b), the op- 

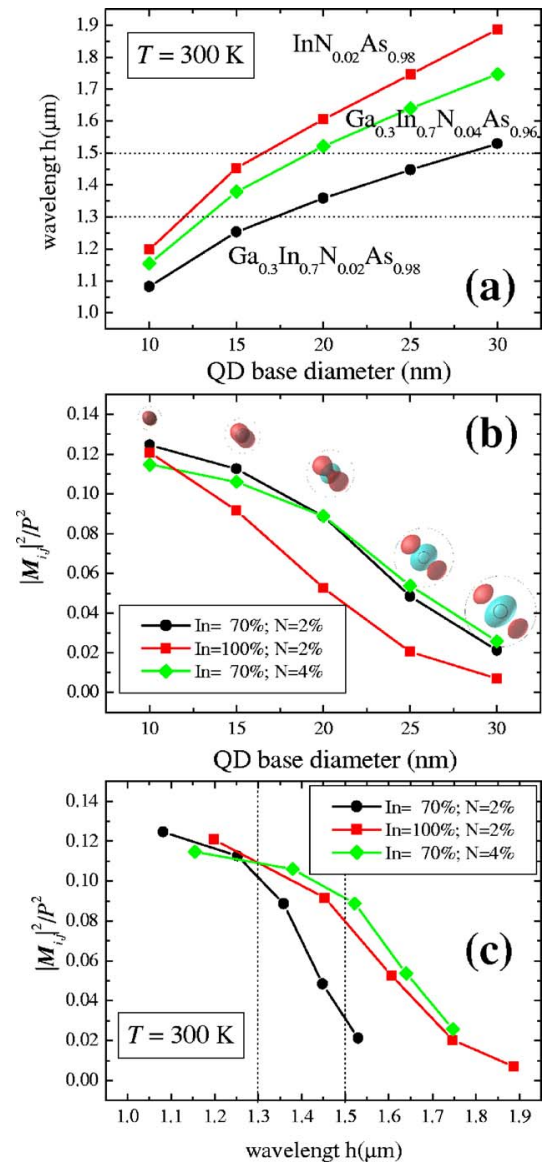

FIG. 2. (Color online) (a) Variation of the fundamental optical transition wavelength vs QD size: $\mathrm{Ga}_{0.3} \mathrm{In}_{0.7} \mathrm{~N}_{0.02} \mathrm{As}_{0.98}$ (circle), $\mathrm{Ga}_{0.3} \mathrm{In}_{0.7} \mathrm{~N}_{0.04} \mathrm{As}_{0.96}$ (diamond), and $\operatorname{InN}_{0.02} \mathrm{As}_{0.98}$ (squares) at $T=300 \mathrm{~K}$. (b) The optical matrix element dependence on the QD size for the three material systems as in (a). (Inset) Evolution of the $e 0$ to $h 0$ charge densities overlaps with the QD size in $\mathrm{Ga}_{0.3} \mathrm{In}_{0.7} \mathrm{~N}_{0.02} \mathrm{As}_{0.98}$ QD. (c) The optical matrix element dependence on the ground state transition wavelength for three material systems obtained from (a) and (b).

tical matrix element variation with the QD size is presented for the three systems. For the $\mathrm{Ga}_{0.3} \mathrm{In}_{0.7} \mathrm{~N}_{0.04} \mathrm{As}_{0.96}$ and $\mathrm{Ga}_{0.3} \mathrm{In}_{0.7} \mathrm{~N}_{0.02} \mathrm{As}_{0.98}$ QDs the optical matrix element is very similar over the whole range of the QD sizes considered. The regions where it is slightly larger in one material than in another are mainly determined by the interplay of the amount of nitrogen character $\left|s_{\mathrm{N}}\right\rangle$ in the ground state electron wave function, $e 0$, confinement, and piezoelectric field, as described above. For intermediate QD sizes the $\mathrm{InN}_{0.02} \mathrm{Ga}_{0.98}$ QD's optical matrix element is significantly (up to $40 \%$ ) smaller than in the other two systems. This is mainly due to a larger piezoeffect that reduces the $e 0-h 0$ overlap. Combining the results from Figs. 2(a) and 2(b) and using the QD size as a parameter, we deduce the variation of the optical matrix element versus wavelength of the $e 0-h 0$ transition. At $1.3 \mu \mathrm{m}$ all three materials exhibit very similar optical characteristics. In this region the small QD size in all three cases provide good quantum confinement, which dominates the optical characteristics. At $1.5 \mu \mathrm{m}$ and above, the $\mathrm{Ga}_{0.3} \mathrm{In}_{0.7} \mathrm{~N}_{0.04} \mathrm{As}_{0.96}$ QDs are clearly the best candidate. Even though larger QD sizes are required than in $\mathrm{InN}_{0.02} \mathrm{As}_{0.98}$ material for the same wavelength, the reduced piezoelectric field due reduced In and increased $\mathrm{N}$ content has less ability to deteriorate optical characteristics. This is another confirmation that an appropriate combination of $\mathrm{N}$ and reduced amounts of In can offer better material candidates for the dilute-N QD's RT-optical characteristics than pure InAs:N material. ${ }^{8}$

In summary, we have presented a theoretical analysis of the optical characteristics of the ideal dilute nitrogen GaInNAs/GaAs QDs. At RT it is estimated that all material systems considered can exhibit emission wavelength of $1.5 \mu \mathrm{m}$ and above. We conclude that (a) increased amount of $\mathrm{N}$ character in $e 0$ and reduced piezoelectric filed in the QD can provide a better uniformity of the optical matrix element when the QD shape varies, and (b) with a reduced amount of In and increased amount of $\mathrm{N}(x \sim 4 \%)$ the same or better optical characteristics can be achieved at 1.3 and $1.5 \mu \mathrm{m}$ than with the InAs:N QD material system. Our analysis shows advantages of $\mathrm{Ga}_{0.3} \mathrm{In}_{0.7} \mathrm{~N}_{0.04} \mathrm{As}_{0.96}$ over equivalent InAs:N QD material system in the design of QD devices operating at $1.5 \mu \mathrm{m}$ and at RT.

The author thanks E. P. O'Reilly for useful discussions. Computing resources were provided by CCLRC's e-Science facility.

${ }^{1}$ C. Y. Liu, S. F. Yoon, Z. Z. Sun, and K. C. Yew, Appl. Phys. Lett. 88, 081105 (2006)

${ }^{2}$ C. Y. Liu, S. F. Yoon, Q. Cao, C. Z. Tong, and Z. Z. Sun, Nanotechnology 17, 5627 (2006)

${ }^{3}$ Q. Gao, M. Buda, H. H. Tan, and C. Jagadish, Electrochem. Solid-State Lett. 8, G57 (2005).

${ }^{4}$ Z. Z. Sun, S. F. Yoon, K. C. Yew, B. X. Bo, D. A. Yan, and T. C. Hang, Appl. Phys. Lett. 85, 1469 (2004).

${ }^{5}$ S. Makino, T. Miyamoto, T. Kageyama, N. Nishiyama, F. Koyama, and K. Iga, J. Cryst. Growth 221, 561 (2000).

${ }^{6}$ W. Shan, W. Walukiewicz, J. W. Ager III, E. E. Haller, J. F. Geisz, D. J. Friedman, J. M. Olson, and S. R. Kurtz, Phys. Rev. Lett. 82, 1221 (1999).

${ }^{7}$ M. Kondow, K. Uomi, K. Hosomi, and T. Mozume, Jpn. J. Appl. Phys., Part 2 33, L1056 (1994).

${ }^{8}$ M. Sopanen, H. P. Xin, and C. W. Tu, Appl. Phys. Lett. 76, 994 (2000).

${ }^{9}$ A. Nishikawa, Y. G. Hong, and C. W. Tu, Phys. Status Solidi B 240, 310 (2003).

${ }^{10}$ A. Nishikawa, Y. G. Hong, and C. W. Tu, J. Vac. Sci. Technol. B 22, 1515 (2004).

${ }^{11}$ A. Nishikawa, R. Katayama, K. Onabe, Y. G. Hong, and C. W. Tu, J. Cryst. Growth 278, 244 (2005).

${ }^{12}$ R. Oshima and Y. Okada, Thin Solid Films 464-465, 229 (2004).

${ }^{13}$ K. C. Yew, S. F. Yoon, and Z. Z. Sun, J. Cryst. Growth 271, 8 (2004).

${ }^{14}$ T. Hakkarainen, J. Toivonen, H. Koskenvaara, M. Sopanen, and H. Lipsanen, J. Phys.: Condens. Matter 16, S3009 (2004).

${ }^{15}$ F. Hatami, S. M. Kim, H. B. Yuen, and J. S. Harris, Appl. Phys. Lett. 89, 133115 (2006)

${ }^{16}$ A. M. Mintairov, T. H. Kosel, J. L. Merz, P. A. Blagnov, A. S. Vlasov, V. M. Ustinov, and R. E. Cook, Phys. Rev. Lett. 87, 277401 (2001).

${ }^{17}$ O. Schumann, S. Birner, M. Baudach, L. Geelhaar, H. Eisele, L. Ivanova, R. Timm, S. Lenz, S. K. Becker, M. Povolotskyi, M. Dahne, G. Abstreiter, and H. Riechert, Phys. Rev. B 71, 245316 (2005).

${ }^{18}$ M. Richter, B. Damilano, J.-Y. Duboz, J. Massies, and A. D. Wieck, Appl. Phys. Lett. 88, 231902 (2006).

${ }^{19}$ C. Pryor, Phys. Rev. B 57, 7190 (1998).

${ }^{20}$ O. Stier, M. Grundmann, and D. Bimberg, Phys. Rev. B 59, 5688 (1999).

${ }^{21}$ S. Tomić, E. P. O'Reilly, P. J. Klar, H. Grüning, W. Heimbrodt, W. M. Chen, and I. A. Buyanova, Phys. Rev. B 69, 245305 (2004).

${ }^{22}$ S. Tomić and E. P. O'Reilly, Phys. Rev. B 71, 233301 (2005).

${ }^{23}$ P. R. C. Kent and A. Zunger, Phys. Rev. Lett. 86, 2613 (2001).

${ }^{24}$ A. Lindsay and E. P. O'Reilly, Phys. Rev. Lett. 93, 196402 (2004).

${ }^{25}$ S. Tomić, Phys. Rev. B 73, 125348 (2006).

${ }^{26}$ S. Tomić, E. P. O'Reilly, R. Fehse, S. J. Sweeney, A. R. Adams, A. D. Andreev, S. A. Choulis, T. J. C. Hosea, and H. Riechert, IEEE J. Sel. Top. Quantum Electron. 9, 1228 (2003).

${ }^{27}$ A. D. Andreev and E. P. O'Reilly, Appl. Phys. Lett. 87, 213106 (2005).

${ }^{28}$ The $\left|d_{e, h h}\right|^{2}$ is reduced to $20 \%$ of its maximal value at $k_{y}=0.1 \AA^{-1}$ in $\mathrm{Ga}_{0.3} \mathrm{In}_{0.7} \mathrm{~N}_{0.04} \mathrm{As}_{0.98}$ vs $k_{y}=0.14 \AA^{-1}$ in $\mathrm{Ga}_{0.3} \mathrm{In}_{0.7}$ As. 